\title{
TET2 is involved in DNA hydroxymethylation, cell proliferation and inflammatory response in keratinocytes
}

\author{
XINXIN LIU $^{1 *}$, XIN WANG $^{2 *}$, NIAN LIU $^{1 *}$, KE ZHU $^{1}$, SONG ZHANG $^{1}$, XIAORU DUAN $^{1}$, \\ YUQIONG HUANG $^{1}$, ZILIN JIN $^{1}$, HIMANSHU JAYPAUL ${ }^{1}$, YAN WU ${ }^{1}$ and HONGXIANG CHEN ${ }^{1}$ \\ ${ }^{1}$ Department of Dermatology, Union Hospital, Tongji Medical College, Huazhong University of Science and Technology, \\ Wuhan, Hebei 430022; ${ }^{2}$ Department of Dermatology, Affiliated Hospital of Nantong University, \\ Nantong, Jiangsu 226001, P.R. China
}

Received July 4, 2019; Accepted January 29, 2020

DOI: $10.3892 / \mathrm{mmr} .2020 .10989$

\begin{abstract}
DNA methylation and hydroxymethylation are the most common epigenetic modifications associated with the cell cycle and the inflammatory response. The present study aimed to investigate the role of 5-hydroxymethyl-cytosine (5-hmC) and ten-eleven translocation-2 (TET2) in keratinocytes. Following TET2 knockdown, dot blot analysis was performed to assess the levels of 5-hmC in keratinocytes, using $\mathrm{HaCaT}$ cells. Subsequently, the viability and cell cycle of HaCaT cells were assessed by MTT, Cell Counting Kit- 8 assay and flow cytometric assays. Cyclin-dependent kinase inhibitor $2 \mathrm{~A}$ and proinflammatory cytokine protein and mRNA expression levels were also detected. The present results suggested that TET2 may play an important role in regulating cellular proliferation by mediating DNA hydroxymethylation in $\mathrm{HaCaT}$ cells. In addition, TET 2 knockdown decreased the production of proinflammatory cytokines, including lipocalin 2, S100 calcium binding protein A7, matrix metallopeptidase $9, \mathrm{C}-\mathrm{X}-\mathrm{C}$ motif chemokine ligand 1, interferon regulatory factor 7 and interleukin-7 receptor. The present study suggested that TET2 regulated cell viability, apoptosis and the expression of inflammatory mediators in keratinocytes. Collectively, the results indicated that TET2 knockdown may relieve inflammatory responses in the skin.
\end{abstract}

Correspondence to: Professor Yan Wu or Professor Hongxiang Chen, Department of Dermatology, Union Hospital, Tongji Medical College, Huazhong University of Science and Technology, 1277 Jiefang Avenue, Wuhan, Hebei 430022, P.R. China

E-mail:wuyan5151@126.com

E-mail: hongxiangchen@hotmail.com

${ }^{*}$ Contributed equally

Key words: ten-eleven translocation-2, keratinocytes, hydroxymethylation, proliferation, inflammation

\section{Introduction}

Epigenetic processes, including DNA methylation, histone modification and non-coding RNA regulation, are inheritable changes that can affect gene expression without altering the DNA sequence (1). In mammals, methylation at the carbon 5 position of cytosine [5-methyl-cytosine $(5 \mathrm{mC})]$ is the most common DNA modification, and it is involved in various biological processes, including cell differentiation, development and proliferation (2). $5 \mathrm{mC}$ is associated with the regulation of a number of pathophysiological processes and states, including gene expression regulation, genomic imprinting, $\mathrm{X}$-chromosome inactivation, repression of transposable elements, embryonic development and cell differentiation (2-5).

The ten-eleven translocation (TET) family, which consists of $\mathrm{Fe}(\mathrm{II})$ - and 2-oxoglutarate-dependent dioxygenases, has attracted increasing attention due to its key role in the reversal of DNA methylation (3). It has been reported that TET proteins (TET1, TET2 and TET3) sequentially oxidize $5 \mathrm{mC}$ to 5-hydroxymethylcytosine (5-hmC), 5-formylcytosine $(5 \mathrm{fC})$ and 5-carboxylcytosine (5caC) in DNA $(2,6,7)$. Previous studies have reported that $5 \mathrm{fC}$ and $5 \mathrm{caC}$ may be converted into cytosine by a thymine-DNA glycosylase/base excision repair-dependent pathway (8-10). In addition to mediating the formation of 5-hmC, TET proteins are involved in gene transcription repression via histone de-acetylation, which represents a DNA methylation-independent epigenetic mechanism (11).

Increasing evidence suggested that $5-\mathrm{hmC}$, which is regulated by TET2, plays an important role in various diseases, such as immune system disorders, hematopoietic diseases and cancers $(5,6,8)$. Li et al (12), reported a predominant role for TET2 in the differentiation of adult neural stem cells by increasing the levels of 5-hmC. Sun et al (13), indicated that inhibiting the TET2 protein decreases the expression of genes associated with cell death by mediating the formation of 5-hmC, which aggravated ischemic damage in a rat model of spinal cord injury. In addition, decreased 5-hmC levels are associated with the progression and poor survival of numerous malignancies, including melanoma, renal cell carcinoma, and gastric and ovarian cancer (14). 
The skin provides the first line of defense against skin injury and invading pathogens, and keratinocytes are the main active cell type in the epidermis $(15,16)$. Recent studies indicated that keratinocytes actively regulate cutaneous immune reactions, which may play a role in inflammatory diseases affecting the skin, including psoriasis and atopic dermatitis $(15,17)$. The present study suggested that TET2 knockdown in HaCaT keratinocytes promoted inflammation. Therefore, the effects of TET2 on epigenetic modifications and cellular functions were investigated in the present study. The results of the present study indicated that the TET2 protein-mediated formation of 5-hmC increased the proportion of apoptotic cells and the expression of inflammatory factors in $\mathrm{HaCaT}$ cells.

\section{Materials and methods}

Cell culture. The human keratinocyte HaCaT cell line (cat. no. GDC106; China Center for Type Culture Collection) was identified by STR profiling performed by Cobioer Biosciences Co., Ltd. HaCaT cells were maintained in minimal essential medium (HyClone; GE Healthcare Life Sciences) supplemented with 10\% FBS (Gibco; Thermo Fisher Scientific, Inc.) and $1 \%$ penicillin-streptomycin (HyClone; GE Healthcare Life Sciences) at $37^{\circ} \mathrm{C}$ with $5 \% \mathrm{CO}_{2}$ in a humidified incubator. The medium was replaced every 3 days. Keratinocytes from generations 3 to 4 were used for subsequent experiments. At $60-80 \%$ confluency, cells were transfected. For small interfering (si)RNA transfection, $\mathrm{HaCaT}$ cells were randomly divided into four groups: i) Negative control (NC) group (transfected with control siRNA); ii) si-TET2-01 group (transfected with a siRNA targeting TET2); iii) si-TET2-02 group (transfected with a second siRNA targeting TET2), and iv) si-TET2-03 group (transfected with a third siRNA targeting TET2). For plasmid DNA transfection, HaCaT cells were randomly divided into two groups: i) NC group (transfected with the empty GV230 vector), and ii) TET2 group (transfected with GV230 containing the coding sequence of TET2).

siRNA transfection. siRNAs were designed and obtained from Guangzhou RiboBioCo.,Ltd. The sequences of the siRNAs were as follows: si-TET2-01, 5'-CCAGAATAGTCGTGTGAGT-3'; si-TET2-02, 5'-GCTCTGA ACGGTATTTAAA-3'; si-TET2-03, 5'-CGAGACTCATAATGTCCAA-3'; and NC siRNA, 5'-UUCUCCGAACGUGUCACGU-3'. TET2 and control siRNAs $(40 \mathrm{pmol} / \mathrm{ml})$ were transfected into $\mathrm{HaCaT}$ cells $\left(5 \times 10^{5} / \mathrm{ml}\right)$ using Lipofectamine ${ }^{\circledR} 3000$ (Invitrogen; Thermo Fisher Scientific, Inc.), according to the manufacturer's protocol. After $48 \mathrm{~h}$, at $37^{\circ} \mathrm{C}$ in a humidified atmosphere with $5 \% \mathrm{CO}_{2}$, cells were collected and used for subsequent experiments.

Plasmid DNA transfection. The TET2 coding sequence was synthesized according to the NM_017628 transcript and inserted into the GV230 plasmid (GeneChem, Inc.). At $70 \%$ confluency, HaCaT cells $\left(5 \times 10^{5} / \mathrm{ml}\right)$ were transfected with the GV230 plasmids ( $1 \mu \mathrm{g} / \mathrm{ml})$ using Lipofectamine 3000 . The empty GV230 vector was used as the negative control. After $48 \mathrm{~h}$, at $37^{\circ} \mathrm{C}$ in a humidified atmosphere with $5 \% \mathrm{CO}_{2}$, cells were collected and used for subsequent experiments.
Dot blot analysis. Genomic DNA from transfected HaCaT cells was extracted using a DNeasy Blood and Tissue kit (cat. no. 69504; Qiagen, Inc.), according to the manufacturer's protocol. DNA concentration (50 $\mathrm{ng} / \mu \mathrm{l})$ was quantified using a micro ultraviolet-visible spectrophotometer. DNA denaturation was performed at $95^{\circ} \mathrm{C}$ for $10 \mathrm{~min}$. Denatured DNA samples were immediately placed in an ice water bath for $5 \mathrm{~min}$. Subsequently, samples $(2 \mu \mathrm{l})$ were spotted on a nylon membrane and blocked with 5\% skimmed milk for $1 \mathrm{~h}$ at room temperature. The membranes were incubated with anti-5-hmC primary antibody (cat. no. 39769; 1:10,000; Active Motif, Inc.) overnight at $4^{\circ} \mathrm{C}$. After washing, the membrane was incubated with a horseradish peroxidase-conjugated secondary antibody (anti-rabbit IgG; 1:2,000; cat. no. 7074; Cell Signaling Technology, Inc.) for $1 \mathrm{~h}$ at room temperature. Detection was performed using a chemiluminescence kit (cat. no. G2014; Wuhan Servicebio Technology Co., Ltd.).

Cell Counting Kit-8 (CCK-8) assay. HaCaT cells $\left(5 \times 10^{5} / \mathrm{ml}\right)$ were cultured in 96-well plates and transfected with plasmid or siRNA for $48 \mathrm{~h}$ at $37^{\circ} \mathrm{C}$ in a humidified atmosphere with $5 \% \mathrm{CO}_{2}$. Cell viability was assessed using the CCK-8 kit (cat. no. WC0037; Wuhan Servicebio Technology Co., Ltd.), according to the manufacturer's protocol. CCK-8 solution was added to each well and the plates were incubated for $1-4 \mathrm{~h}$ at $37^{\circ} \mathrm{C}$. The absorbance was measured at a wavelength of $450 \mathrm{~nm}$ using a microplate reader. The assay was performed in triplicate.

MTT assay. HaCaT cells $\left(5 \times 10^{5} / \mathrm{ml}\right)$ were cultured in 96-well plates and transfected with plasmid DNA or siRNA for $48 \mathrm{~h}$ at $37^{\circ} \mathrm{C}$ in a humidified atmosphere with $5 \% \mathrm{CO}_{2}$. Cell viability was measured using an MTT assay (cat. no. G4101; Wuhan Servicebio Technology Co., Ltd.), according to the manufacturer's protocol. MTT solution was added to each well and incubated for $4 \mathrm{~h}$ at $37^{\circ} \mathrm{C}$ in a humidified atmosphere with $5 \% \mathrm{CO}_{2}$. Subsequently, the supernatant was removed and DMSO was used to dissolve the purple formazan. The absorbance of each well was measured at a wavelength of $570 \mathrm{~nm}$ using a microplate reader. Cell viability was calculated as the ratio of absorbance at a wavelength of $570 \mathrm{~nm}$ between each group and the control group.

Flow cytometric analysis. Following transfection, HaCaT cells $\left(5 \times 10^{5} / \mathrm{ml}\right)$ were cultured for $48 \mathrm{~h}$ at $37^{\circ} \mathrm{C}$ in a humidified atmosphere with $5 \% \mathrm{CO}_{2}$. Subsequently, cells were trypsinized (0.25\% trypsin at $37^{\circ} \mathrm{C}$ for $\left.3-5 \mathrm{~min}\right)$, harvested and washed twice with cold PBS. To measure apoptosis, the FITC Annexin V Apoptosis Detection kit (cat. no. 556547; BD Biosciences) was used, according to the manufacturer's protocol. After incubation for $15 \mathrm{~min}$ at room temperature, the samples were analyzed by flow cytometry (BD ${ }^{\mathrm{TM}}$ LSR-II; BD Biosciences). Data analysis was performed using FlowJo software (v10.5.3; Becton, Dickinson and Company). The apoptotic rate was the sum of the early and late apoptotic rates.

For the analysis of the cell cycle, cells were fixed in $70 \%$ pre-cooled ethanol at $4^{\circ} \mathrm{C}$ overnight. Subsequently, the cells were stained with propidium iodide (PI)/RNase staining buffer (cat. no. 550825; BD Biosciences) for $15 \mathrm{~min}$ at room 
A

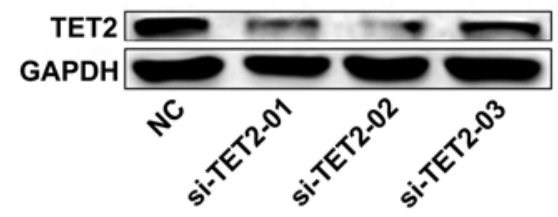

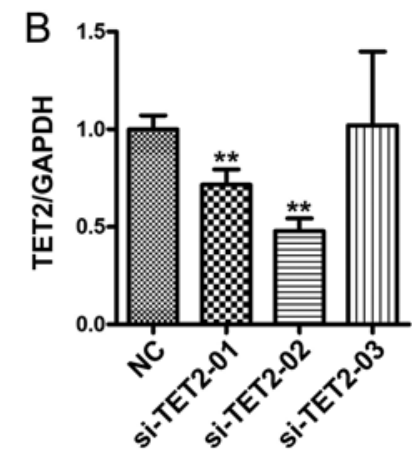

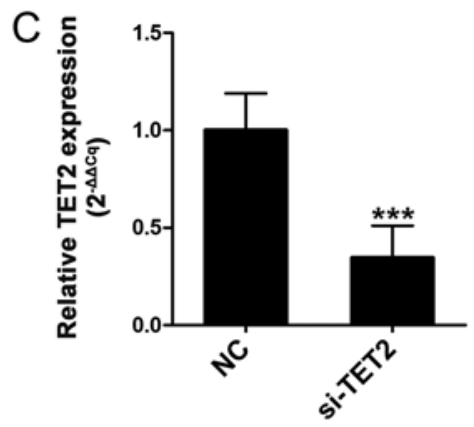

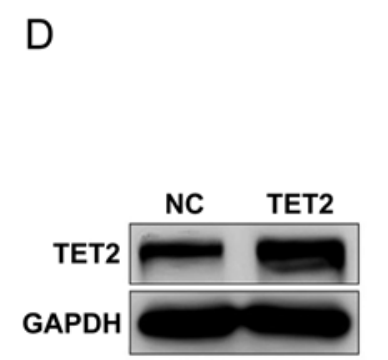

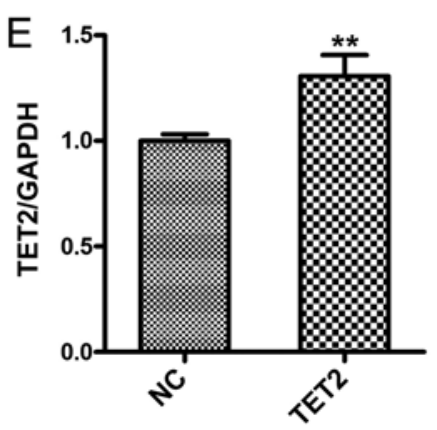

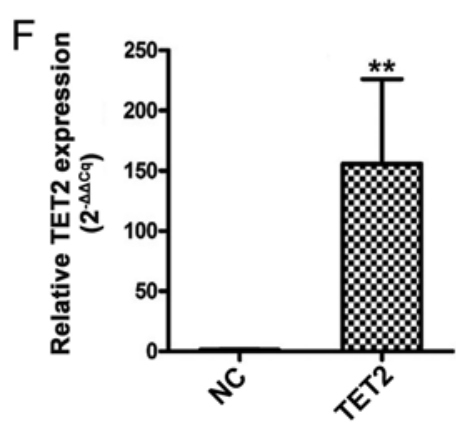

G

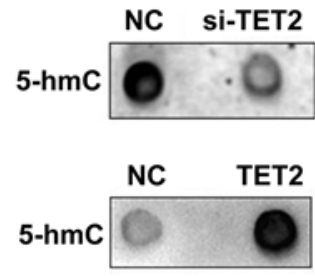

Figure 1. TET2 modulates the levels of 5-hmC in HaCaT cells. Protein levels of TET2 were (A) determined by western blotting and (B) quantified following transfection with three specific siRNAs. (C) mRNA expression levels of TET2 were analyzed by reverse transcription-quantitative PCR in HaCaT cell transfected with si-TET2 or si-NC. Protein levels of TET2 were (D) determined by western blotting and (E) quantified following transfection with TET2-overexpression plasmid or NC plasmid. (F) mRNA expression levels of TET2 following transfection with TET-2 overexpression plasmid or NC plasmid. (G) Dot blot analyses for 5-hmC were performed following TET2 knockdown or overexpression in HaCaT cells. Data are presented as the mean \pm SD of three independent experiments performed in triplicate. ${ }^{* *} \mathrm{P}<0.01,{ }^{* * *} \mathrm{P}<0.001$ vs. NC. TET2, ten-eleven translocation-2; 5-hmC, 5-hydroxymethylcytosine; si/siRNA, small interfering RNA; NC, negative control.

temperature and analyzed using a FACScan flow cytometer (Becton, Dickinson and Company), according to the manufacturer's instructions. Data analysis was performed using FlowJo software (v10.5.3; Becton, Dickinson and Company).

Reverse transcription-quantitative PCR (RT-qPCR). Total RNA was extracted from HaCaT cells using TRIzol ${ }^{\circledR}$ reagent (cat. no. 15596-026; Invitrogen; Thermo Fisher Scientific, Inc.), according to the manufacturer's protocol. RNA $(1 \mu \mathrm{g})$ was reverse transcribed into cDNA using the PrimeScript RT Reagent kit (cat. no. RR036A; Takara Biotechnology Co., Ltd.), according to the manufacturer's protocols. Subsequently, qPCR was performed using a real-time PCR detection system and TB Green ${ }^{\circledR}$ Premix Ex Taq ${ }^{\mathrm{TM}}$ (cat. no. RR420A; Takara Biotechnology Co., Ltd.). The thermocycling conditions were: Initial denaturation at $95^{\circ} \mathrm{C}$ for $10 \mathrm{~min}$, followed by 40 cycles of $95^{\circ} \mathrm{C}$ for $15 \mathrm{sec}, 60^{\circ} \mathrm{C}$ for $1 \mathrm{~min}$ and $72^{\circ} \mathrm{C}$ for $3 \mathrm{~min}$. The primers used for qPCR are presented in Table SI. mRNA levels were quantified using the $2^{-\Delta \Delta \mathrm{Cq}}$ method (18) and normalized to GAPDH.

Western blotting. HaCaT cells were lysed using RIPA cell lysis buffer (cat. no. P0013B; Beyotime Institute of Biotechnology), and the supernatant was collected by centrifugation at $13,000 \mathrm{x}$ for $5 \mathrm{~min}$ at $4^{\circ} \mathrm{C}$. Total protein was quantified using a BCA protein assay kit (cat. no. G2026; Wuhan Servicebio Technology Co., Ltd.). Equal amounts of protein $(30 \mu \mathrm{g})$ were separated by $10 \%$ SDS-PAGE and then transferred to PVDF membranes. After blocking with 5\% non-fat milk for $1 \mathrm{~h}$ at room temperature, the membranes were incubated overnight at $4^{\circ} \mathrm{C}$ with primary antibodies targeted against: GAPDH (cat. no. 118; 1:1,000; Cell Signaling Technology, Inc.), TET2 (cat. no. ab94580; 1:1,000; Abcam), cyclin-dependent kinase inhibitor 2A (P16 ${ }^{\mathrm{INK} 4 \mathrm{a}}$; cat. no. ab108349; 1:2,000; Abcam), interferon regulatory factor 7 (IRF7; cat. no. 22392-1-AP; 1:2,000; ProteinTech Group, Inc.), S100 calcium binding protein A7 (S100A7; cat. no. 13061-1-AP; 1:500; ProteinTech Group, Inc.), matrix metallopeptidase 9 (MMP9; cat. no. 10375-2-AP; 1:1,000; ProteinTech Group, Inc.), lipocalin 2 (LCN2; cat. no. 26991-1-AP; 1:1,000; ProteinTech Group, Inc.), C-X-C motif chemokine ligand 1 (CXCL1; cat. no. A5802; 1:1,000; ABclonal Biotech Co., Ltd.) and interleukin-7 receptor (IL7R; cat. no. 17626-1-AP; 1:2,000; ProteinTech Group, Inc.). Subsequently, the membranes were incubated for $1 \mathrm{~h}$ at room temperature with corresponding horseradish peroxidase-conjugated secondary antibodies (anti-rabbit or anti-mouse IgG; cat. nos. 7074 and 7076; 1:2,000; Cell Signaling Technology, Inc.). Protein bands were visualized using an ECL kit (cat. no. G2014; Wuhan Servicebio Technology Co., Ltd.) and GelCapture (v7.0; DNR Bio-Imaging Systems, Inc.), according to the manufacturer's instructions. Protein expression was quantified using ImageJ software (v1.8.0; National Institutes of Health) with GAPDH as the loading control. 

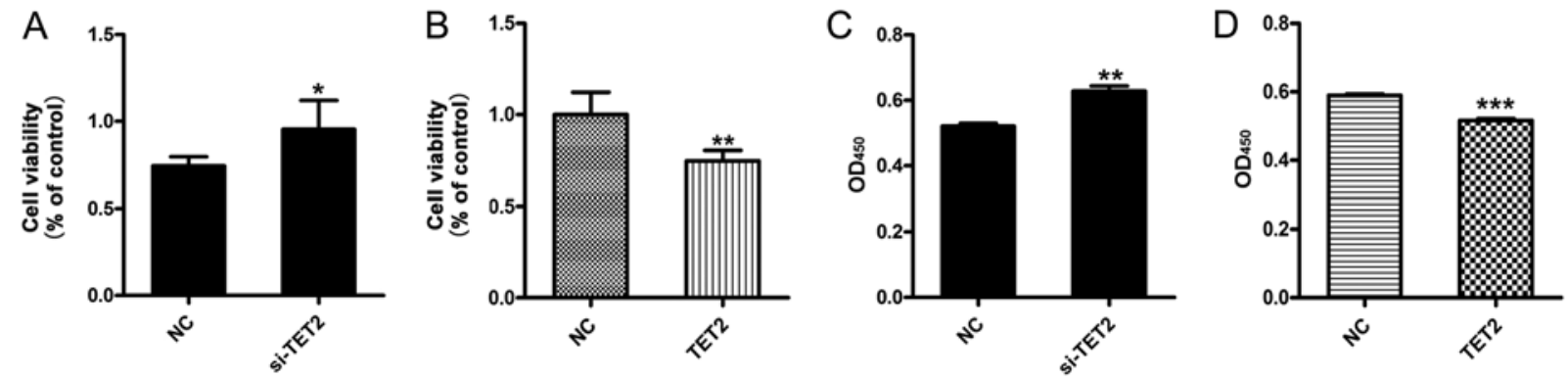

Figure 2. TET2 knockdown or overexpression affects the viability of HaCaT cells. Cell viability was measured using an MTT assay following (A) TET2 knockdown or (B) overexpression in $\mathrm{HaCaT}$ cells. Cell viability was calculated as the ratio of absorbance at a wavelength of $570 \mathrm{~nm}$ between each group and the control group. Cell viability was also determined using the Cell Counting Kit-8 assay following (C) TET2 knockdown or (D) overexpression in HaCaT cells Data are presented as the mean $\pm \mathrm{SD}$ of three independent experiments performed in triplicate. ${ }^{*} \mathrm{P}<0.05,{ }^{* *} \mathrm{P}<0.01,{ }^{* * *} \mathrm{P}<0.001$ vs. NC group. TET2, ten-eleven translocation-2; NC, negative control; OD, optical density; si, small interfering RNA.

Statistical analysis. Data are presented as the mean \pm SD. Statistical analyses were performed using GraphPad Prism software (v6.0; GraphPad Software, Inc.). Comparisons between data containing 2 groups were performed using an unpaired two-tailed Student's t-test. Comparisons between data containing $>2$ groups were performed using one-way ANOVA followed by Tukey's post hoc test. $\mathrm{P}<0.05$ was considered to indicate a statistically significant difference.

\section{Results}

Effect of TET2 on the level of 5-hmC in HaCaT cells. To investigate whether the levels of genomic 5-hmC are associated with TET2 protein levels in HaCaT cells, three siRNAs targeting TET2 were designed. TET2 knockdown efficiency was evaluated by western blotting (Fig. 1A and B). Compared with the control group, two siRNAs targeting TET2 significantly decreased TET 2 mRNA expression; siRNA-TET2-02 was the most efficient in reducing TET2 mRNA and protein expression (Fig. 1A-C). Therefore, siRNA-TET2-02 was selected for further experiments. Subsequently, TET2 overexpression was performed, and transduction efficiency was confirmed by western blotting and RT-qPCR (Fig. 1D-F). Previous studies have reported that members of the TET family are able to reverse DNA methylation patterns by converting 5 -mC into 5 -hmC $(2,6,7)$. Therefore, a dot blot analysis was performed to test the levels of 5-hmC following TET2 overexpression. The results suggested that 5-hmC expression was notably altered following TET 2 knockdown or overexpression, compared with the control group (Fig. 1G).

Effect of TET2 on HaCaT cell viability and apoptosis. The viability of HaCaT cells following TET2 knockdown or overexpression was measured. The MTT assay results suggested that si-TET2 significantly increased $\mathrm{HaCaT}$ cell viability (Fig. 2A) and TET2 overexpression significantly decreased HaCaT cell viability (Fig. 2B). In addition, the CCK-8 assay was performed to further examine cell viability, and the results were consistent with the MTT assay (Fig. 2C and D). To examine the effects of TET2 protein on HaCaT cell apoptosis, a quantitative analysis of cell apoptosis was performed by Annexin V-PI staining. The results indicated that the proportion of apoptotic cells was significantly decreased in
$\mathrm{HaCaT}$ cells transfected with si-TET2 compared with the control group (Fig. 3A and B), whereas TET2 overexpression significantly increased the proportion of apoptotic $\mathrm{HaCaT}$ cells (Fig. 3C and D). The results suggested that TET2 may play an important role in regulating $\mathrm{HaCaT}$ cell proliferation.

Effect of TET2 on the cell cycle in HaCaT cells. To further investigate the effects of TET2 protein on HaCaT cell proliferation, flow cytometric analyses were conducted to assess the cell cycle profile. Following TET2 knockdown, a significantly decreased proportion of cells was observed in the G0/G1 phase, and a significantly increased proportion of cells was observed in the $\mathrm{S}$ and $\mathrm{G} 2 / \mathrm{M}$ phases, compared with the control group (Fig. 4A and B). By contrast, TET2 overexpression displayed the opposite effect on the cell cycle in HaCaT cells (Fig. 4C and D). To further examine the effects of TET2 on the cell cycle, $\mathrm{P} 16^{\mathrm{INK} 4 \mathrm{a}}$ protein and mRNA expression levels were detected by western blotting and RT-qPCR, respectively. Compared with the control group, TET2 knockdown significantly decreased the protein and mRNA expression levels of P16 ${ }^{\text {INK4a }}$ (Fig. 4E-G); however, TET2 overexpression increased P16 ${ }^{\text {INK4a }}$ protein expression compared with the control group (Fig. 4H and I). mRNA expression levels of P16 $6^{\mathrm{INK} 4 \mathrm{a}}$ were increased in $\mathrm{HaCaT}$ cells following TET2 overexpression, but the difference was not statistically significant (Fig. 4J). The results indicated that TET2 may inhibit $\mathrm{HaCaT}$ cell proliferation by regulating cell cycle-related proteins, such as $\mathrm{P} 16^{\mathrm{INK} 4 \mathrm{a}}$.

Effect of TET2 on the expression levels of proinflammatory cytokines in HaCaT cells. It has been reported that epigenetic modifications may influence inflammatory mediators in chronic inflammatory diseases, including psoriasis (19). Our previous integrated bioinformatics analysis identified various hub genes that were methylated in psoriatic tissues, including LCN2, S100A7, MMP9, CXCL1, IRF7 and IL7R (20). To further investigate the function of TET2 during the inflammatory response in $\mathrm{HaCaT}$ cells, the expression levels of the aforementioned proinflammatory cytokines were investigated by RT-qPCR and western blotting (Fig. 5A-N). The expression of various inflammation-related cytokines was significantly decreased in the si-TET2 group compared with control group (Fig. 5A-F, M and N). TET2 overexpression significantly increased the expression of inflammation-related cytokines 


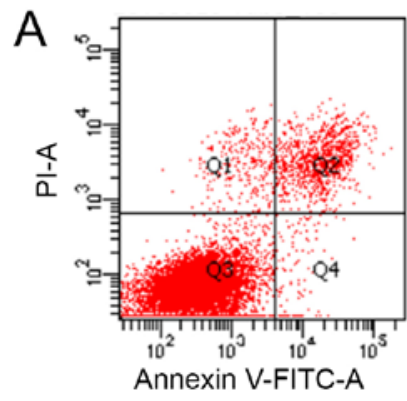

NC

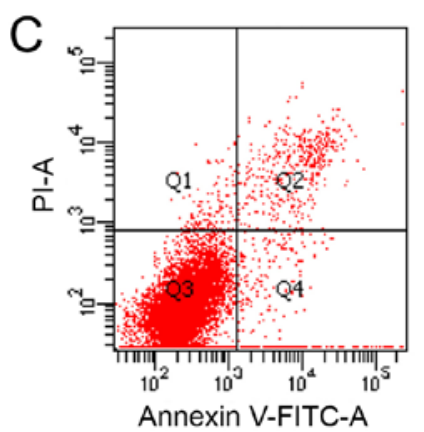

NC
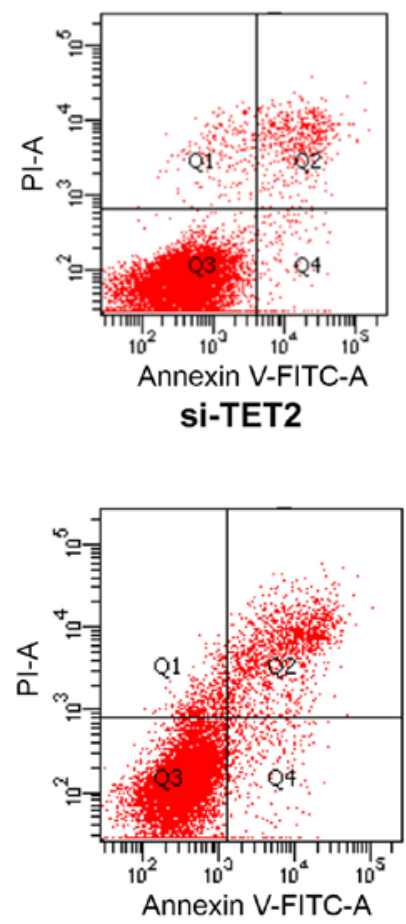

TET2
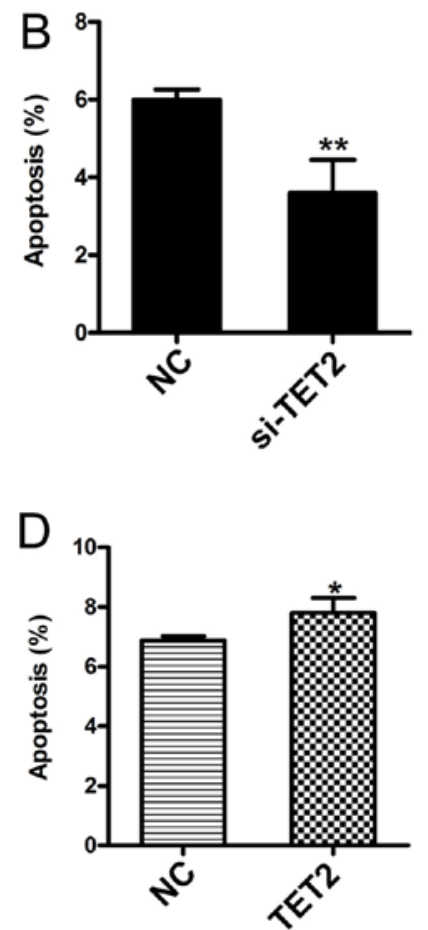

Figure 3. Alterations to the proportion of apoptotic HaCaT cells in response to TET2 knockdown or overexpression. Proportion of apoptotic cells was (A) determined by flow cytometry and (B) quantified following TET2 knockdown in HaCaT cells. Proportion of apoptotic cells was (C) determined by flow cytometry and (D) quantified following TET2 overexpression in HaCaT cells. Data are presented as the mean \pm SD of three independent experiments performed in triplicate. ${ }^{*} \mathrm{P}<0.05,{ }^{* *} \mathrm{P}<0.01$ vs. NC group. TET2, ten-eleven translocation-2; NC, negative control; PI, propidium iodide; si, small interfering RNA.

compared with the control group (Fig. 5G-L). The results suggested that TET2 acted as a transcriptional regulator of the proinflammatory response in $\mathrm{HaCaT}$ cells.

\section{Discussion}

The epigenetic activity of TET2 in a number of hematologic malignancies, and metabolic and autoimmune diseases has been investigated (21-23). However, the role of TET2 in skin disease remains unclear. Our previous study reported that 5-hmC and TET2 levels were increased in epidermal keratinocytes in psoriasiform dermatitis (24). To further investigate the function of TET2 in inflammatory skin diseases, the present study performed experiments using the keratinocyte $\mathrm{HaCaT}$ cell line. Cell apoptosis, cell cycle and proinflammatory cytokine expression were detected following TET2 knockdown or overexpression in $\mathrm{HaCaT}$ cells. The results suggested that TET2 expression was positively associated with $5-\mathrm{hmC}$ levels and the proportion of apoptotic cells, but negatively associated with cell viability and cell cycle progression. Furthermore, TET2 knockdown in $\mathrm{HaCaT}$ cells decreased the expression of several proinflammatory cytokines, including LCN2, S100A7, MMP9, CXCL1, IRF7 and IL7R. The data indicated that TET2 epigenetically regulated the expression of inflammatory factors in keratinocytes.

DNA methylation, the most common epigenetic regulation, is partly controlled by DNA methyltransferases (DNMTs), including DNMT1 and DNMT3A/3B $(23,25)$. The levels and patterns of DNA methylation are regulated by two contrasting processes, methylation and demethylation, which have an essential role in gene expression regulation (2). Since the crystal structure of TET 2 and the 5mC-DNA complex were reported, knowledge of the mechanisms underlying TET-mediated demethylation has gradually improved $(4,9)$. 5 -hmC is an important intermediate product of DNA demethylation, which is downregulated following TET2 knockdown $(3,5,7,26)$. Dot blot analysis indicated that TET 2 knockdown or overexpression decreased or increased 5-hmC expression levels, respectively. The results of the present study were in line with previous studies $(5,7)$, thus suggesting that DNA hydroxymethylation patterns in HaCaT cells are regulated by TET 2 .

TET2 affects cellular proliferation and cell cycle progression in various cell lines, such as $\mathrm{CD} 4^{+} \mathrm{T}$ and Hela cells, in other conditions, including autoimmune diseases and development (5,27-30). However, the precise mechanism underlying TET2 in HaCaT cells remains unclear. The results suggested that $\mathrm{HaCaT}$ viability was increased following TET 2 knockdown and the proportion of apoptotic cells decreased. Conversely, TET2 overexpression induced opposing effects. The results suggested that TET2 may be involved in regulating $\mathrm{HaCaT}$ cell proliferation. Furthermore, the flow cytometric analysis indicated that TET2 regulated cell proliferation by inducing cell cycle arrest at the G0/G1 phase. Similar results have been reported in two TET2 inducible cell lines (Ba/F3-EPOR and UT7) (30). A limitation of the present study was that cell proliferation was not analyzed using direct assays, for example 5-bromo-2-deoxyuridine (BrdU) labeling. However, Prikrylova et al (27) reported that TET2-induced 5-hmC expression was inversely proportional to the proportion of apoptotic cells in HeLa cell lines using the BrdU assay, which 


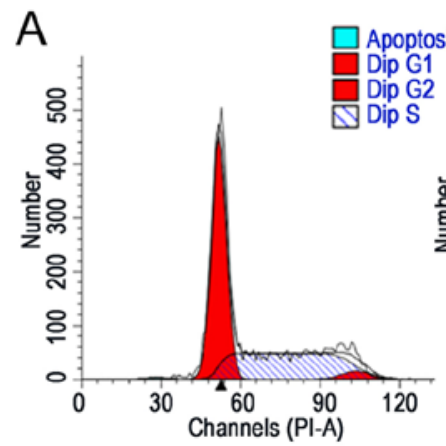

NC

C

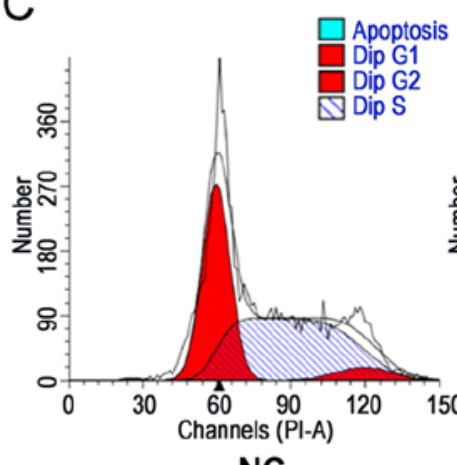

NC

E

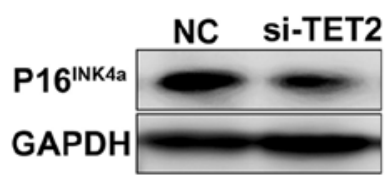

$\mathrm{H}$

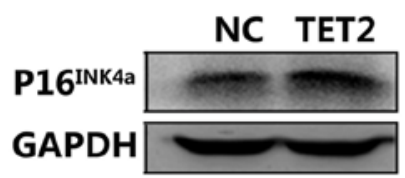

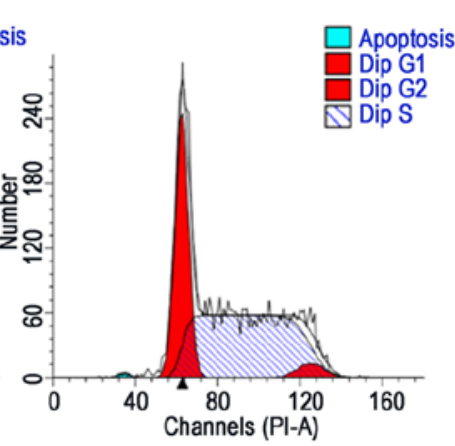

Si-TET2
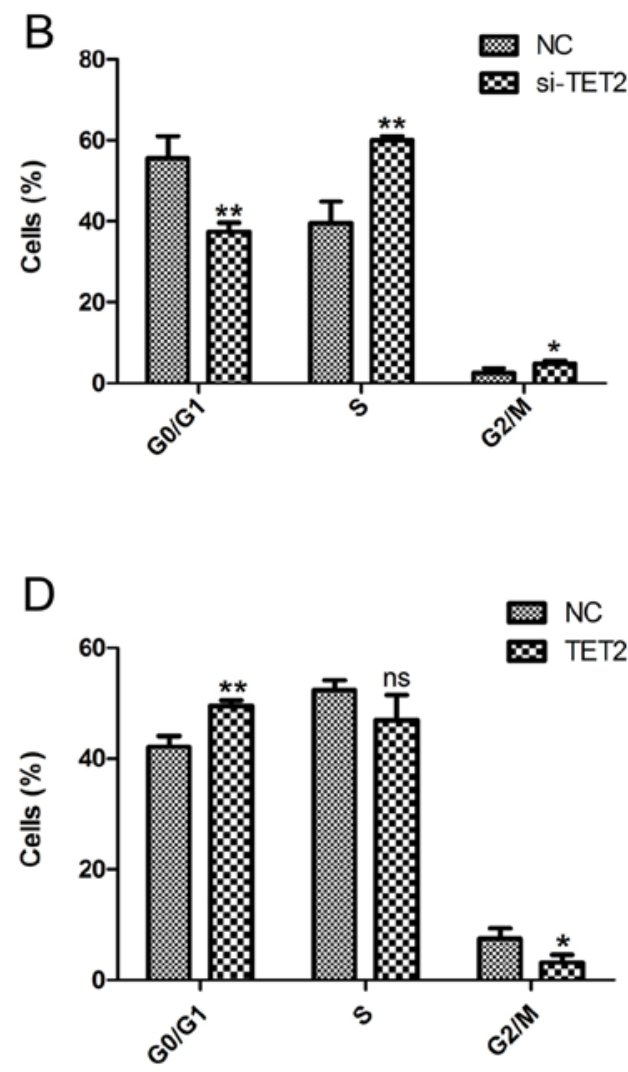

TET2
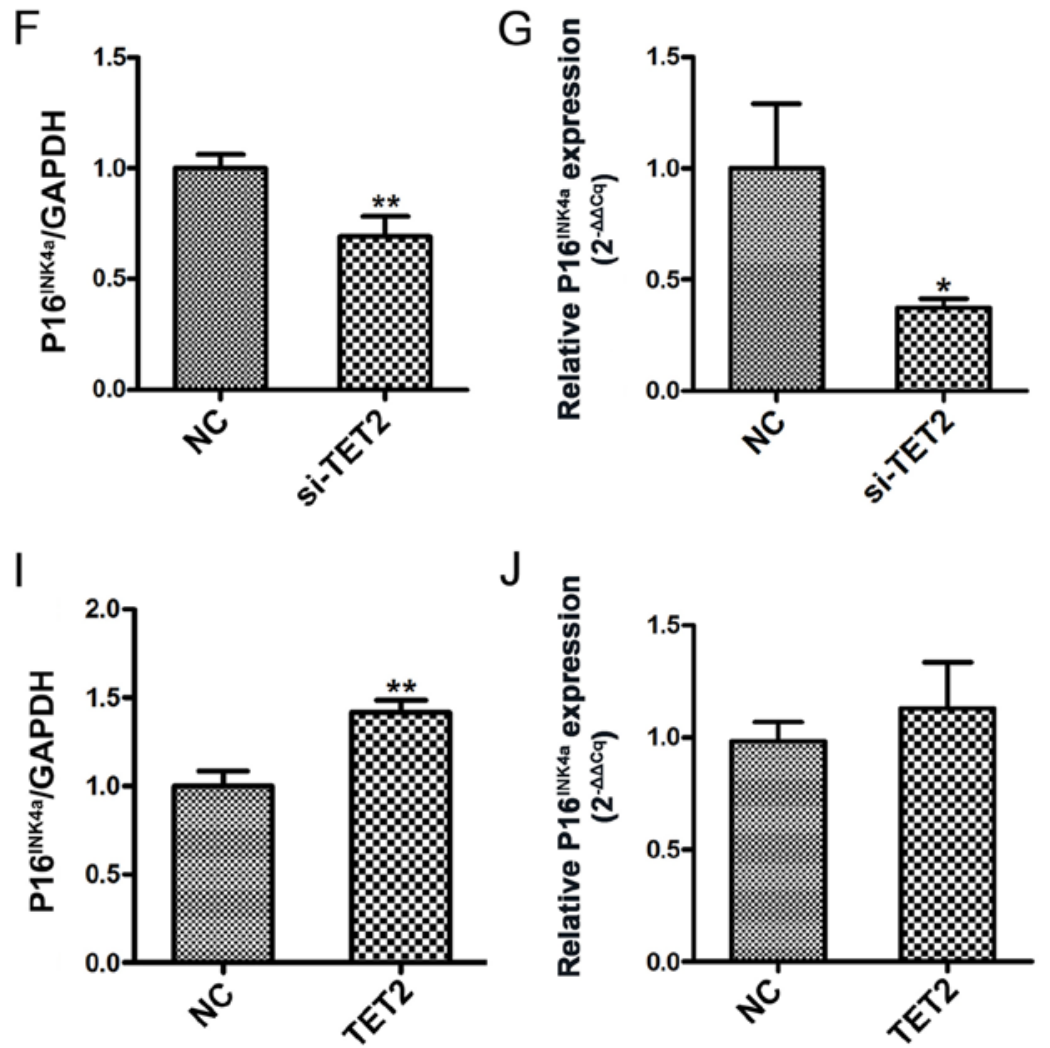

Figure 4. TET2 expression alters the expression of the cell cycle-associated protein P16 ${ }^{\mathrm{INK} 4 \mathrm{a}}$ in $\mathrm{HaCaT}$ cells. Cell cycle profile of $\mathrm{HaCaT}$ cells transfected with si-TET2 was (A) determined by flow cytometry and (B) quantified. Cell cycle profile of HaCaT cells transfected with TET2-overexpression plasmid was (C) determined by flow cytometry and (D) quantified. P16 $6^{\text {INK4a }}$ protein levels were (E) determined by western blotting and (F) quantified following TET2 knockdown in HaCaT cells. (G) P16 $6^{\text {INK4a }}$ mRNA levels following TET2 knockdown in HaCaT cells were determined by RT-qPCR. P16 ${ }^{\text {INK4a }}$ protein levels were $(\mathrm{H})$ determined by flow cytometry and (I) quantified following TET2 overexpression in HaCaT cells. (J) P16 ${ }^{\text {INK4a }}$ mRNA levels following TET2 overexpression in $\mathrm{HaCaT}$ cells were determined by RT-qPCR. Data are presented as the mean $\pm \mathrm{SD}$ of three independent experiments performed in triplicate. ${ }^{*} \mathrm{P}<0.05,{ }^{* *} \mathrm{P}<0.01$ vs. NC group. TET2, ten-eleven translocation-2; P16 ${ }^{\mathrm{INK} 4 \mathrm{a}}$, cyclin-dependent kinase inhibitor 2A; si, small interfering RNA; RT-qPCR, reverse transcription-quantitative PCR; NC, negative control; PI, propidium iodide; ns, not significant. 
A

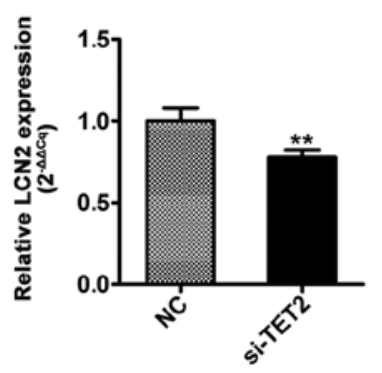

$\mathrm{E}$

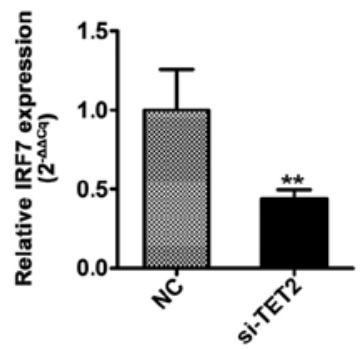

I

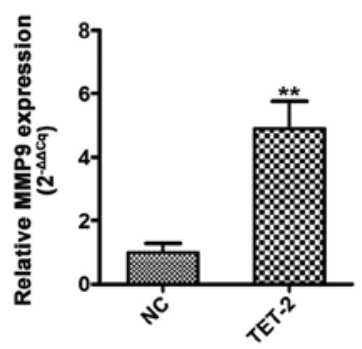

M

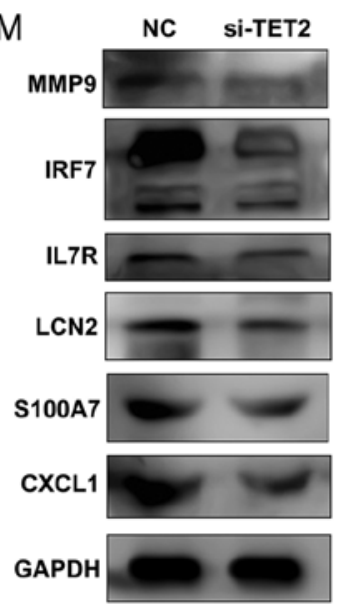

B

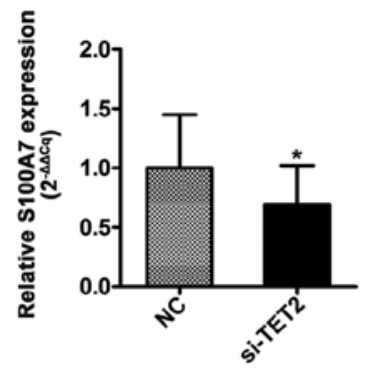

$\mathrm{F}$

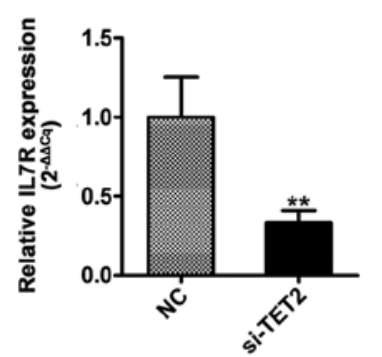

$\mathrm{J}$

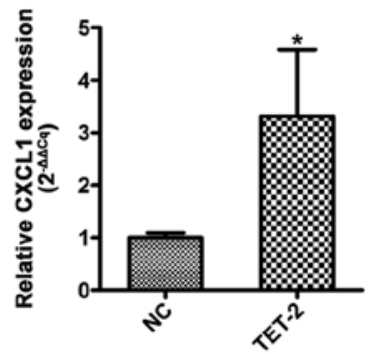

C

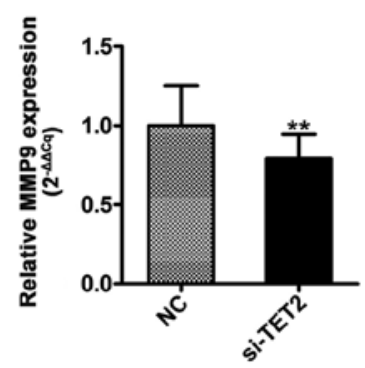

G

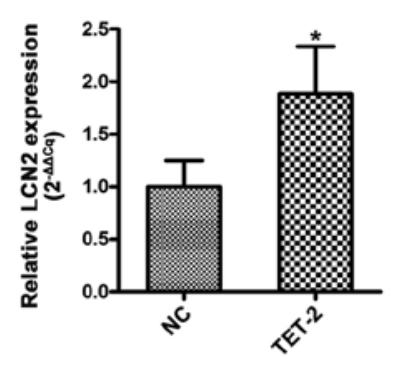

$\mathrm{K}$

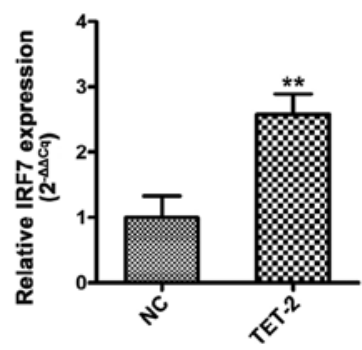

$\mathrm{N}$
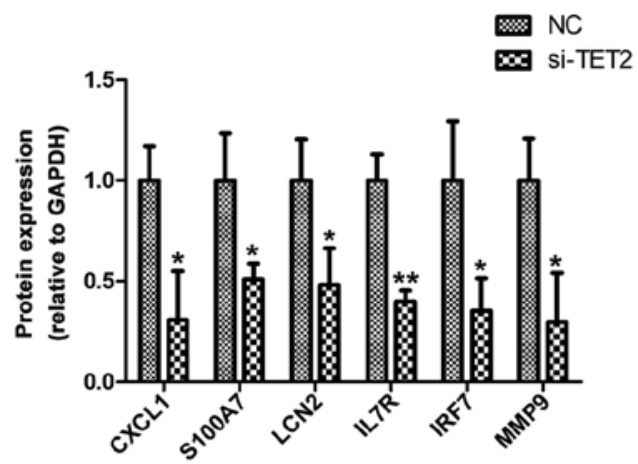

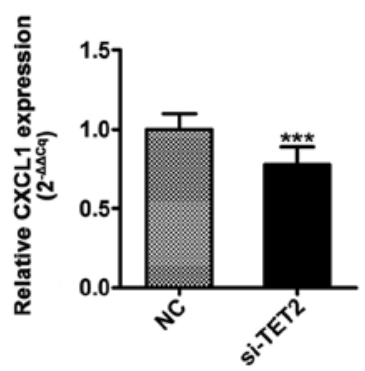

$\mathrm{H}$

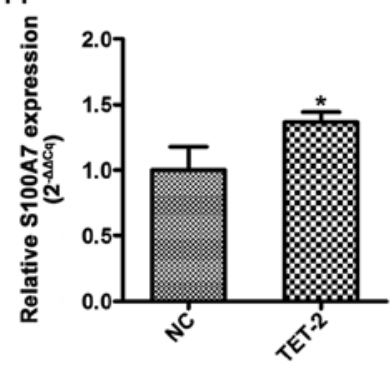

L

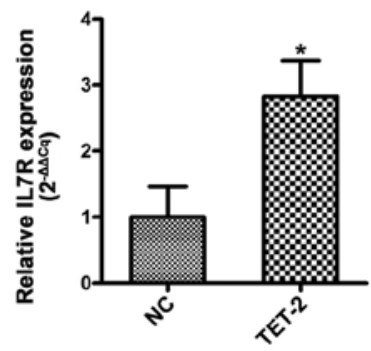

Figure 5. TET2 regulates the expression levels of proinflammatory cytokines at the mRNA and protein level in HaCaT cells. RT-qPCR was performed to determine the mRNA expression levels of (A) LCN2, (B) S100A7, (C) MMP9, (D) CXCL1, (E) IRF7 and (F) IL7R following TET2 knockdown in HaCaT cells. RT-qPCR was performed to determine the mRNA expression levels of (G) LCN2, (H) S100A7, (I) MMP9, (J) CXCL1, (K) IRF7 and (L) IL7R following TET2 overexpression in $\mathrm{HaCaT}$ cells. Protein levels of proinflammatory cytokines were (M) determined by western blotting and (N) quantified following TET2 knockdown in HaCaT cells. Data are presented as the mean $\pm \mathrm{SD}$ of three independent experiments performed in triplicate. ${ }^{*} \mathrm{P}<0.05,{ }^{* *} \mathrm{P}<0.01,{ }^{* * *} \mathrm{P}<0.001 \mathrm{vs}$ NC group. TET2, ten-eleven transloation-2; RT-qPCR, reverse transcription-quantitative PCR; LCN2, lipocalin 2; S100A7, S100 calcium binding protein A7; MMP9, matrix metallopeptidase 9; CXCL1, C-X-C motif chemokine ligand 1; IRF7, interferon regulatory factor 7; IL7R, interleukin-7 receptor; NC, negative control; si, small interfering RNA.

is in line with the results of the present study. Collectively, the results suggested that TET2 may influence cell cycle arrest and cell proliferation. To further investigate the underlying mechanism, whether TET2-induced cell cycle arrest was mediated by the cell cycle-associated protein $\mathrm{P} 16^{\mathrm{INK4a}}$, an inhibitor of cyclin-dependent kinase 4 (31), was investigated. 
It has been reported that $\mathrm{P} 16^{\mathrm{INK} 4 \mathrm{a}}$ affects cell proliferation and differentiation by negatively regulating the cell cycle (32). Recently, several studies have reported that TET 2 regulates cell cycle-associated proteins in neural and trophoblast stem cells $(28,29)$. In line with these previous studies, the results of the present study suggested that the increase in P16 ${ }^{\text {INK4a }}$ expression was mediated by TET2 overexpression, and si-TET2 decreased the level of P16 $6^{\mathrm{INK} 4 \mathrm{a}}$ expression. The results indicated that P16 ${ }^{\mathrm{INK} 4 \mathrm{a}}$ upregulation was decreased following TET2 knockdown.

Epigenetic factors are involved in various chronic inflammatory diseases, including type 2 diabetes, Alzheimer's disease and inflammatory bowel diseases $(19,33)$. DNA methylation and hydroxymethylation may be involved in inflammation by altering the homeostasis of various inflammatory mediators, such as interleukin (IL)-6, TNF receptor associated factor 6 and IL-23 (19). Lagos et al (34), reported that DNA methylation and hydroxymethylation were associated with glandular inflammation and dysfunction, which might be a novel target for the treatment of Sjögren's syndrome. Wang et al (35), revealed that TET2 knockdown by siRNAs not only affected the level of MyD88 innate immune signal transduction adaptor hydroxymethylation, but also inhibited lipopolysaccharide-induced inflammation in human dental pulp cells. Our previous study suggested that TET 2 modulates the expression of proinflammatory cytokines in a mouse model of psoriasiform dermatitis (24). In the present study, the effects of DNA demethylation on inflammatory factors were examined in vitro. The results of the present study suggested that the expression levels of proinflammatory mediators, including LCN2, S100A7, MMP9, CXCL1, IRF7 and IL7R, may be positively associated with the level of DNA hydroxymethylation. Therefore, the results suggested that epigenetic changes may affect the progression of inflammatory skin diseases. The present study investigated the mechanism underlying TET2 in HaCaT cells; however, characterization of the hydroxymethylation pattern of specific genes in keratinocytes requires further investigation.

The present study investigated the potential role and mechanism underlying the effect of TET2 on cell proliferation and expression levels of various inflammation mediators in $\mathrm{HaCaT}$ cells. The results indicated that the regulation of the cell cycle and expression of proinflammatory cytokines was mediated by DNA hydroxymethylation. Further investigation is required to assess whether TET2 protein plays an important role in the pathogenesis of inflammatory skin diseases.

\section{Acknowledgements}

Not applicable.

\section{Funding}

The present study was supported by the National Natural Science Foundation of China (grant nos. 81673057 and 81502730).

\section{Availability of data and materials}

The datasets used and analyzed during the present study are available from the corresponding author on reasonable request.

\section{Authors' contributions}

$\mathrm{XL}, \mathrm{NL}, \mathrm{YW}$ and $\mathrm{HC}$ wrote the manuscript. XL, XW, NL, $\mathrm{KZ}, \mathrm{SZ}, \mathrm{XD}, \mathrm{YH}, \mathrm{HJ}$ and $\mathrm{ZJ}$ performed the experiments and collected the data. XL, XW, NL, KZ, YW and HC designed the study. HJ, YW and HC edited the manuscript for English language. All authors read and approved the final manuscript.

\section{Ethics approval and consent to participate}

Not applicable.

\section{Patient consent for publication}

Not applicable.

\section{Competing interests}

The authors declare that they have no competing interests.

\section{References}

1. Shen J,Abu-Amer Y,O'Keefe RJ and McAlinden A: Inflammation and epigenetic regulation in osteoarthritis. Connect Tissue Res 58: 49-63, 2017.

2. Wu X and Zhang Y: TET-mediated active DNA demethylation: Mechanism, function and beyond. Nat Rev Genet 18: 517-534, 2017.

3. Li D, Guo B, Wu H, Tan L and Lu Q: TET family of dioxygenases: Crucial roles and underlying mechanisms. Cytogenet Genome Res 146: 171-180, 2015.

4. Hu L, Li Z, Cheng J, Rao Q, Gong W, Liu M, Shi YG, Zhu J, Wang P and $\mathrm{Xu}$ Y: Crystal structure of TET2-DNA complex: Insight into TET-mediated 5mC oxidation. Cell 155: 1545-1555, 2013.

5. Ichiyama K, Chen T, Wang X, Yan X, Kim BS, Tanaka S, Ndiaye-Lobry D, Deng Y, Zou Y, Zheng P, et al: The methylcytosine dioxygenase Tet 2 promotes DNA demethylation and activation of cytokine gene expression in T cells. Immunity 42: 613-626, 2015.

6. Pastor WA, Aravind L and Rao A: TETonic shift: Biological roles of TET proteins in DNA demethylation and transcription. Nat Rev Mol Cell Biol 14: 341-356, 2013.

7. Ito S, Shen L, Dai Q, Wu SC, Collins LB, Swenberg JA, He C and Zhang Y: Tet proteins can convert 5-methylcytosine to 5-formylcytosine and 5-carboxylcytosine. Science 333: 1300-1303, 2011.

8. Rasmussen KD and Helin K: Role of TET enzymes in DNA methylation, development, and cancer. Genes Dev 30: 733-750, 2016.

9. He YF, Li BZ, Li Z, Liu P, Wang Y, Tang Q, Ding J, Jia Y, Chen Z, $\mathrm{Li}$ L, et al: Tet-mediated formation of 5-carboxylcytosine and its excision by TDG in mammalian DNA. Science 333: 1303-1307, 2011.

10. Weber AR, Krawczyk C, Robertson AB, Kuśnierczyk A, Vågbø CB, Schuermann D, Klungland A and Schär P: Biochemical reconstitution of TET1-TDG-BER-dependent active DNA demethylation reveals a highly coordinated mechanism. Nat Commun 7: 10806, 2016.

11. Zhang Q, Zhao K, Shen Q, Han Y, Gu Y, Li X, Zhao D, Liu Y, Wang C,Zhang X, et al: Tet2 is required to resolve inflammation by recruiting Hdac2 to specifically repress IL-6. Nature 525 : 389-393, 2015.

12. Li X, Yao B, Chen L, Kang Y, Li Y, Cheng Y, Li L, Lin L, Wang Z, Wang M, et al: Ten-eleven translocation 2 interacts with forkhead box $\mathrm{O} 3$ and regulates adult neurogenesis. Nat Commun 8: 15903, 2017.

13. Sun H, Miao Z, Wang H, Tao Y, Yang J, Cai J, Wang J and Wang Y: DNA hydroxymethylation mediated traumatic spinal injury by influencing cell death-related gene expression. J Cell Biochem 119: 9295-9302, 2018.

14. Uchiyama R, Uhara H, Uchiyama A, Ogawa E, Takazawa Y, Ashida A, Koga H, Hayashi K, Kiniwa Y and Okuyama R: 5-Hydroxymethylcytosine as a useful marker to differentiate between malignant melanomas and benign melanocytic nevi. J Dermatol Sci 73: 161-163, 2014. 
15. Benhadou F, MintoffD and Del Marmol V: Psoriasis: Keratinocytes or immune cells - Which is the trigger? Dermatology 235: 91-100, 2019.

16. Bitschar K, Wolz C, Krismer B, Peschel A and Schittek B: Keratinocytes as sensors and central players in the immune defense against Staphylococcus aureus in the skin. J Dermatol Sci 87: 215-220, 2017.

17. Asahina R and Maeda S: A review of the roles of keratinocyte-derived cytokines and chemokines in the pathogenesis of atopic dermatitis in humans and dogs. Vet Dermatol 28: 16-e5, 2017.

18. Livak KJ and Schmittgen TD: Analysis of relative gene expression data using real-time quantitative PCR and the 2(-Delta Delta C(T)) Method. Methods 25: 402-408, 2001.

19. Fogel O, Richard-Miceli $\mathrm{C}$ and Tost J: Epigenetic changes in chronic inflammatory diseases. Adv Protein Chem Struct Biol 106: 139-189, 2017.

20. Wang X, Liu X, Liu N and Chen H: Prediction of crucial epigenetically-associated, differentially expressed genes by integrated bioinformatics analysis and the identification of S100A9 as a novel biomarker in psoriasis. Int J Mol Med 45: 93-102, 2020.

21. Poole CJ, Lodh A, Choi JH and van Riggelen J: MYC deregulates TET1 and TET2 expression to control global DNA (hydroxy) methylation and gene expression to maintain a neoplastic phenotype in T-ALL. Epigenetics Chromatin 12: 41, 2019.

22. Chiba S: Dysregulation of TET2 in hematologic malignancies. Int J Hematol 105: 17-22, 2017.

23. Raghuraman S, Donkin I, Versteyhe S, Barrès R and Simar D: The emerging role of epigenetics in inflammation and immunometabolism. Trends Endocrinol Metab 27: 782-795, 2016.

24. Wang X, Liu X, Duan X, Zhu K, Zhang S, Gan L, Liu N, Jaypaul H, Makamure JT, Ming Z, et al: Ten-eleven translocation-2 regulates DNA hydroxymethylation status and psoriasiform dermatitis progression in mice. Acta Derm Venereol 98: 585-593, 2018.

25. Cheng $X$ and Blumenthal RM: Mammalian DNA methyltransferases: A structural perspective. Structure 16: 341-350, 2008.

26. Ko M, An J, Bandukwala HS, Chavez L, Aijö T, Pastor WA, Segal MF, Li H, Koh KP, Lähdesmäki H, et al: Modulation of TET2 expression and 5-methylcytosine oxidation by the CXXC domain protein IDAX. Nature 497: 122-126, 2013.
27. Prikrylova T, Robertson J, Ferrucci F, Konorska D, Aanes H, Manaf A, Zhang B, Vågbø CB, Kuśnierczyk A, Gilljam KM, et al: 5-hydroxymethylcytosine marks mammalian origins acting as a barrier to replication. Sci Rep 9: 11065, 2019.

28. Chrysanthou S, Senner CE, Woods L, Fineberg E, Okkenhaug H, Burge S, Perez-Garcia V and Hemberger M: A critical role of TET1/2 proteins in cell-cycle progression of trophoblast stem cells. Stem Cell Reports 10: 1355-1368, 2018

29. Shimozaki K: Ten-eleven translocation 1 and 2 confer overlapping transcriptional programs for the proliferation of cultured adult neural stem cells. Cell Mol Neurobiol 37: 995-1008, 2017.

30. Mahfoudhi E, Talhaoui I, Cabagnols X, Della Valle V, Secardin L, Rameau P, Bernard OA, Ishchenko AA, Abbes S, Vainchenker W, et al: TET2-mediated 5-hydroxymethylcytosine induces genetic instability and mutagenesis. DNA Repair (Amst) 43: 78-88, 2016.

31. O'Neill CJ and McCluggage WG: p16 expression in the female genital tract and its value in diagnosis. Adv Anat Pathol 13: 8-15, 2006.

32. Aagaard L, Lukas J, Bartkova J, Kjerulff AA, Strauss M and Bartek $\mathrm{J}$ : Aberrations of p16Ink4 and retinoblastoma tumour-suppressor genes occur in distinct sub-sets of human cancer cell lines. Int J Cancer 61: 115-120, 1995.

33. Stylianou E: Epigenetics of chronic inflammatory diseases. J Inflamm Res 12: 1-14, 2018.

34. Lagos C, Carvajal P, Castro I, Jara D, González S, Aguilera S, Barrera MJ, Quest AFG, Bahamondes V, Molina C, et al: Association of high 5-hydroxymethylcytosine levels with Ten Eleven Translocation 2 overexpression and inflammation in Sjögren's syndrome patients. Clin Immunol 196: 85-96, 2018.

35. Wang X, Feng Z, Li Q, Yi B and Xu Q: DNA methylcytosine dioxygenase ten-eleven translocation 2 enhances lipopolysaccharide-induced cytokine expression in human dental pulp cells by regulating MyD88 hydroxymethylation. Cell Tissue Res 373: 477-485, 2018.

This work is licensed under a Creative Commons Attribution-NonCommercial-NoDerivatives 4.0 International (CC BY-NC-ND 4.0) License. 